\title{
Distribution of Calcium and Phosphorus in Leaves of the Proteaceae
}

\author{
Peta L. Clode ${ }^{1}$, Patrick Hayes ${ }^{1,2}$, Caio Guilherme Pereira ${ }^{1,2}$, Hans Lambers ${ }^{2}$ \\ ${ }^{1}$ Centre for Microscopy, Characterisation \& Analysis, The University of Western Australia. Crawley, \\ WA 6009 Australia \\ ${ }^{2}$ School of Plant Biology, The University of Western Australia. Crawley, WA 6009 Australia
}

The overall aim of our research program is to discover the physiological basis of calcium toxicity in Proteaceae plant species that typically inhabit low-phosphorus, acidic soils and avoid calcareous (alkaline) soils. The phenomenon of Ca toxicity has been known for decades, but so far no clear mechanistic explanation is available. We propose that $\mathrm{Ca}$ toxicity in P-efficient Proteaceae is the result of a shift in cell type where $\mathrm{P}$ accumulates, from epidermal cells as seen in dicots generally to mesophyll cells in P-efficient Proteaceae species [1]. Conversely, we hypothesise that Ca toxicity is avoided in the few Proteaceae species that inhabit calcareous soils by shifting the accumulation of $\mathrm{Ca}$ from mesophyll cells, where dicots generally accumulate $\mathrm{Ca}$, to epidermal cells so as to prevent interference with efficient $\mathrm{P}$ utilisation.

Leaves of several Banksia spp. growing in different soil environments in both Australia and Chile were dissected and rapid frozen in liquid nitrogen, before being subsequently prepared for cellular elemental analysis and mapping by EDS X-ray microanalysis. Frozen samples were either freeze-substituted in an anhydrous ether:acrolein mixture, resin embedded [2] and microplaned to produce transverse sections of leaf; or simply microplaned transversely while frozen and analysed in the fully frozen-hydrated state [3].

Samples were analysed at CMCA in a Zeiss Supra 55 field emission SEM fitted with a Leica cryostage and an Oxford X-Max80 SDD X-ray detector $\left(80 \mathrm{~mm}^{2}\right)$ interfaced to Oxford Instruments AZtecEnergy software. The microscope was operated at $15 \mathrm{kV}$ in high current mode. Immediately prior to each map acquisition, the instrument was calibrated and the beam current measured and recorded using a pure copper standard. Standards included polished mineral standards and microplaned frozen solutions of aqueous salts at various concentrations $\left(\right.$ e.g. $\left.\mathrm{CaCl}_{2}\right)$. Elemental maps were acquired at a resolution of $1024 \times 768$ pixels, for $>400$ frames with a dwell time of 50-100 $\mu$ s per pixel. Drift correction and pulsepile up correction were activated. Using the Oxford Instruments AZtecEnergy software, quantitative numerical data were subsequently extracted from regions of interest drawn on the element maps, with individual spectra from each pixel summed and processed to yield concentration data. Summed spectra from regions of interest were quantified using the AZtec XPP model for matrix corrections.

Elemental maps of leaf transverse sections are revealing the distribution and concentration of key elements in individual cell types, including the epidermis, hypodermis, sclerenchyma, palisade mesophyll and bundle sheath cells (Figure 1). Preliminary data suggest that $\mathrm{P}$ is concentrated within the mesophyll cells and $\mathrm{Ca}$ within the epidermal cells, as originally hypothesised [4].

\section{References:}

[1] Shane, M. W. Tissue and cellular phosphorus storage during development of phosphorus toxicity in Hakea prostrata (Proteaceae). Journal of Experimental Botany 55, 1033-1044 (2004).

[2] Marshall, A. Freeze-substitution as a preparation technique for biological X-ray microanalysis.

Scanning Electron Microscopy 395-408 (1980). 
[3] Marshall, A. T. \& Clode, P. L. X-ray microanalysis of Rb+ entry into cricket Malpighian tubule cells via putative K+ channels. Journal of Experimental Biology 212, 2977-2982 (2009).

[4] The authors acknowledge ARC Discovery Program funding (DP130100005) and the use of the equipment at CMCA, a facility funded by Universities, and State and Commonwealth governments.
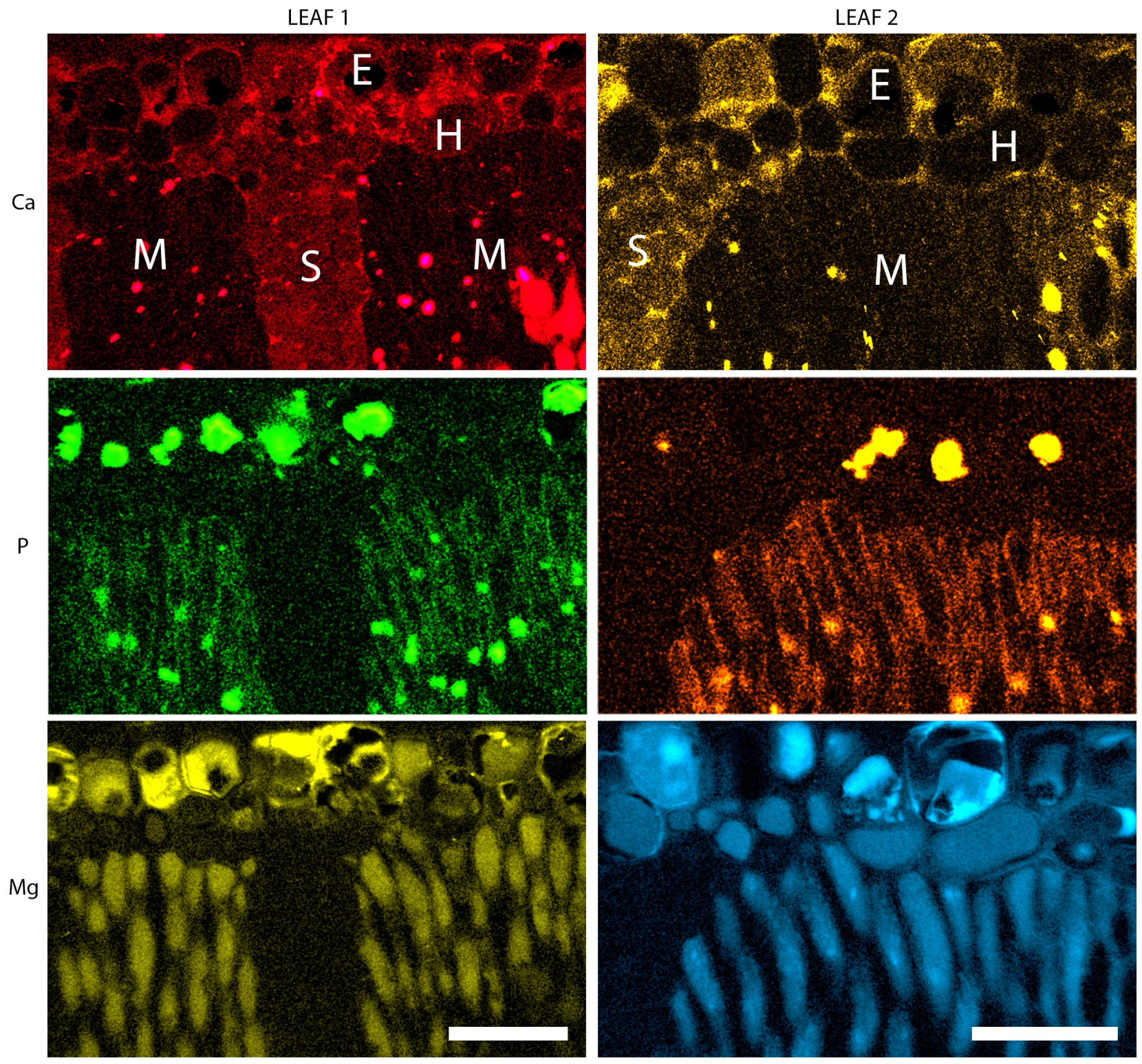

Qualitatitve element maps of freeze-substituted Banksia attenuata leaves. $\mathrm{E}=$ epidermis; $\mathrm{H}=$ hypodermis; $\mathrm{S}=$ sclerenchyma; $\mathrm{M}=$ palisade mesophyll. Scale bars $=50 \mathrm{um}$. 